\title{
VIM for Solving the Pollution Problem of a System of Lakes
}

\author{
J. Biazar, ${ }^{1}$ M. Shahbala, ${ }^{1}$ and H. Ebrahimi ${ }^{2}$ \\ ${ }^{1}$ Department of Mathematics, Faculty of Science, University of Guilan, P.O. Box 41635-19141, P.C. 4193833697, Rasht, Iran \\ ${ }^{2}$ Department of Mathematics, Islamic Azad University (branch: Rasht), P.O. Box 41335-3516, Rasht, Iran
}

Correspondence should be addressed to J. Biazar, biazar@guilan.ac.ir

Received 26 April 2010; Accepted 7 July 2010

Academic Editor: Onur Toker

Copyright ( 2010 J. Biazar et al. This is an open access article distributed under the Creative Commons Attribution License, which permits unrestricted use, distribution, and reproduction in any medium, provided the original work is properly cited.

\begin{abstract}
Pollution has become a very serious threat to our environment. Monitoring pollution is the first step toward planning to save the environment. The use of differential equations of monitoring pollution has become possible. In this paper the pollution problem of three lakes with interconnecting channels has been studied. The variational iteration method has been applied to compute an approximate solution of the system of differential equations, governing on the problem. Three different types of input models: sinusoidal, impulse, and step will be considered for monitoring the pollution in the lakes. The results are compared with those obtained by Adomian decomposition method. This comparison reveals that the variational iteration method is easier to be implemented.
\end{abstract}

\section{Introduction}

He's variational iteration method plays an important role in recent researches in different fields of sciences and engineering. This method is proposed by the Chinese mathematician, $\mathrm{He},[1-3]$ as a modification of the general Lagrange multiplier method. It has been shown to solve efficiently, easily, and accurately a large class of nonlinear problems with approximation converging to accurate solutions. This method has been used to study many problems in different disciplines, hyperbolic equation [4], wave equation [5], nonlinear chemistry problems [6], and quadratic Riccati differential equation [7].

To illustrate its basic idea of the method, we consider the following nonlinear equation:

$$
L u(t)+N u(t)=g(t)
$$

where $L$ is a linear operator, $N$ is a nonlinear operator, and $g(t)$ is a known analytical function.

According to VIM, we can construct the following correction functional:

$$
u_{n+1}(t)=u_{n}(t)+\int_{0}^{t} \lambda\left\{L u_{n}(s)+N \tilde{u}_{n}(s)-g(s)\right\} d s,
$$

where $\lambda$ is a Lagrange multiplier [8], which can be identified optimally via variational theory $[9,10], u_{n}$ is the $n$th approximate solution, and $\tilde{u}_{n}$ denotes a restricted variation [10], that is, $\delta \tilde{u}_{n}=0$.

\section{Modeling of the Pollution Problem}

Figure 1 shows the system of three lakes that are modeled in [11]. Each lake is considered to be a large compartment and the interconnecting channel as pipes between the compartments.

The direction of flow in the channels or pipes is indicated by the arrows in the figure. A pollutant is introduced into the first lake and $p(t)$ denotes the rate at which the pollutant enters the lake per unit time. The function $p(t)$ may be constant or may vary with time. We are interested in knowing the level of pollution in each lake at any time.

Let $x_{i}(t)$ denotes the amount of the pollution in lake $i$ at any time $t \geq 0$, where $i=1,2,3$. We assume the pollutant in each lake to be uniformly distributed throughout the lake by some mixing process, and the volume of water $V_{i}$ in lake $i$ remains constant for each of the lakes. Also we assume that the type of pollution is persistent and not degrading to other forms. Then the concentration of the pollutant in lake $i$ at any time is given by

$$
c_{i}(t)=\frac{x_{i}(t)}{V_{i}}
$$




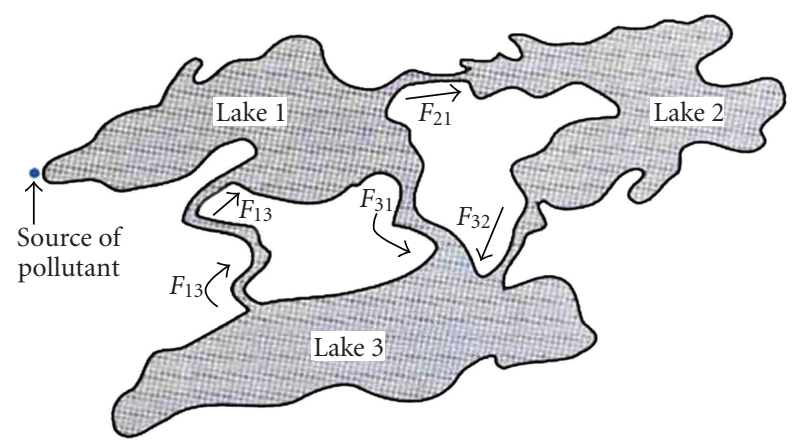

FIGURE 1: System of three lakes with interconnecting channels.

Each lake initially is assumed to be free of any contaminant, so $x_{i}(0)=0$ for each $i=1,2,3$. To model the dynamic behavior of the system of lakes, we let constant $F_{j i}$ denote the flow rate from lake $i$ to lake $j$. The flux of pollutant flowing from lake $i$ into lake $j$ at any time $t$, denoted by $r_{j i}(t)$, is defined by

$$
r_{j i}(t)=F_{j i} c_{i}(t)=\frac{F_{j i}}{V_{i}} x_{i}(t) .
$$

Thus $r_{j i}(t)$ measures the rate at which the concentration of pollutant in lake $i$ flows into lake $j$ at time $t$. We will observe that

rate of change of pollutant $=$ Input rate - output rate.

Applying this principle to each lake results in the following system of first-order equations:

$$
\begin{gathered}
\frac{d x_{1}}{d t}=\frac{F_{13}}{V_{3}} x_{3}(t)+p(t)-\frac{F_{31}}{V_{1}} x_{1}(t)-\frac{F_{21}}{V_{1}} x_{1}(t), \\
\frac{d x_{2}}{d t}=\frac{F_{21}}{V_{1}} x_{1}(t)-\frac{F_{32}}{V_{2}} x_{2}(t) \\
\frac{d x_{3}}{d t}=\frac{F_{31}}{V_{1}} x_{1}(t)+\frac{F_{32}}{V_{2}} x_{2}(t)-\frac{F_{13}}{V_{3}} x_{3}(t)
\end{gathered}
$$

To remain constant the volume of each lake, the flow rate into each lake must balance the flow out of the lake. So we earn the following conditions:

$$
\begin{array}{ll}
\text { Lake 1: } & F_{13}=F_{21}+F_{31}, \\
\text { Lake 2: } & F_{21}=F_{32}, \\
\text { Lake 3: } & F_{31}+F_{32}=F_{13} .
\end{array}
$$

\section{Numerical Results and Comparison with the ADM}

There are three different types of input models used to monitor pollutants in a lake. The input models are the sinusoidal, impulse, and step input.
3.1. Sinusoidal Input. The Sinusoidal input model is used for pollutants that are introduced to the lake periodically. Sinusoidal input changes $p(t)$ to a more useful one

$$
p(t)=p_{i} *\left(1+a * \sin \left(\frac{2 \pi * t}{T}\right)\right) .
$$

The additional variables for the sinusoidal model are defined as follows:

$a$ : the normalize amplitude,

$T$ : the period of fluctuations,

$p_{i}$ : the average input concentration of pollutant.

The actual amplitude of the term is $p_{i}(1+a)$ for the peaks and $p_{i}(1-a)$ for the valleys.

An example would be a manufacturing plant dumping waste producing more output during the day than at night because of hour of operation, hence a periodic input. The concentration of the lake eventually converges to the average input concentration of the contaminant.

Now we assume $p(t)=1+\sin (t)$, and for comparison with the results found by Biazar [11], the following values, for variables, are considered:

$$
\begin{gathered}
V_{1}=2900 \mathrm{mi}^{3}, \quad V_{2}=850 \mathrm{mi}^{3}, \quad V_{3}=1180 \mathrm{mi}^{3}, \\
F_{21}=18 \mathrm{mi}^{3} / \text { year }, \quad F_{32}=18 \mathrm{mi}^{3} / \text { year, } \\
F_{31}=20 \mathrm{mi}^{3} / \text { year, } \quad F_{13}=38 \mathrm{mi}^{3} / \text { year. }
\end{gathered}
$$

So system (6) will be

$$
\begin{gathered}
x_{1}^{\prime}(t)=\frac{38}{1180} x_{3}(t)+(1+\sin (t))-\frac{38}{2900} x_{1}(t), \\
x_{2}^{\prime}(t)=\frac{18}{2900} x_{1}(t)-\frac{18}{850} x_{2}(t), \\
x_{3}^{\prime}(t)=\frac{20}{2900} x_{1}(t)+\frac{18}{850} x_{2}(t)-\frac{38}{1180} x_{3}(t), \\
x_{1}(0)=0, \quad x_{2}(0)=0, \quad x_{3}(0)=0 .
\end{gathered}
$$

According to VIM, we can construct the correction functionals of system (10) as follows:

$$
\begin{aligned}
& x_{1_{n+1}}(t)=x_{1_{n}}(t)+\int_{0}^{t} \lambda_{1}\left\{x_{1_{n}}^{\prime}(\xi)-\frac{38}{1180} \tilde{x}_{3_{n}}(\xi)\right. \\
& \left.-1-\sin (\xi)+\frac{38}{2900} \tilde{x}_{1_{n}}(\xi)\right\} d \xi, \\
& x_{2_{n+1}}(t)=x_{2_{n}}(t)+\int_{0}^{t} \lambda_{2}\left\{x_{2_{n}}^{\prime}(\xi)-\frac{18}{2900} \tilde{x}_{1_{n}}(\xi)\right. \\
& \left.+\frac{18}{850} \tilde{x}_{2_{n}}(\xi)\right\} d \xi, \\
& x_{3_{n+1}}(t)=x_{3_{n}}(t)+\int_{0}^{t} \lambda_{3}\left\{x_{3_{n}}^{\prime}(\xi)-\frac{20}{2900} \tilde{x}_{1_{n}}(\xi)\right. \\
& \left.-\frac{18}{850} \tilde{x}_{2_{n}}(\xi)+\frac{38}{1180} \tilde{x}_{3_{n}}(\xi)\right\} d \xi,
\end{aligned}
$$


where $\lambda_{1}, \lambda_{2}$, and $\lambda_{3}$ are general Lagrange multipliers, $\tilde{x}_{1_{n}}(\xi), \tilde{x}_{2_{n}}(\xi)$, and $\tilde{x}_{3_{n}}(\xi)$ denote restricted variations, that is, $\delta \tilde{x}_{1_{n}}(\xi)=\delta \tilde{x}_{2_{n}}(\xi)=\delta \tilde{x}_{3_{n}}(\xi)=0$.

Making the above correction functional stationary, we have

$$
\begin{aligned}
\delta x_{1_{n+1}}(t)= & \delta x_{1_{n}}(t)+\left.\lambda_{1}(\xi) \delta x_{1_{n}}(\xi)\right|_{0} ^{t} \\
& -\int_{0}^{t} \lambda_{1}^{\prime}(\xi) \delta x_{1_{n}}(\xi) d \xi=0, \\
\delta x_{2_{n+1}}(t)= & \delta x_{2_{n}}(t)+\left.\lambda_{2}(\xi) \delta x_{2_{n}}(\xi)\right|_{0} ^{t} \\
& -\int_{0}^{t} \lambda_{2}^{\prime}(\xi) \delta x_{2_{n}}(\xi) d \xi=0, \\
\delta x_{3_{n+1}}(t)= & \delta x_{3_{n}}(t)+\left.\lambda_{3}(\xi) \delta x_{3_{n}}(\xi)\right|_{0} ^{t} \\
& -\int_{0}^{t} \lambda_{3}^{\prime}(\xi) \delta x_{3_{n}}(\xi) d \xi=0 .
\end{aligned}
$$

The stationary conditions can be obtained as follows:

$$
\begin{aligned}
& \delta x_{1_{n}}:\left\{\begin{array}{l}
1+\left.\lambda_{1}(\xi)\right|_{\xi=t}=0, \\
\lambda_{1}^{\prime}(\xi)=0,
\end{array}\right. \\
& \delta x_{2_{n}}:\left\{\begin{array}{l}
1+\left.\lambda_{2}(\xi)\right|_{\xi=t}=0, \\
\lambda_{2}^{\prime}(\xi)=0,
\end{array}\right. \\
& \delta x_{3_{n}}:\left\{\begin{array}{l}
1+\left.\lambda_{3}(\xi)\right|_{\xi=t}=0, \\
\lambda_{3}^{\prime}(\xi)=0 .
\end{array}\right.
\end{aligned}
$$

Therefore, the Lagrange multipliers can be identified as

$$
\lambda_{1}=\lambda_{2}=\lambda_{3}=-1 \text {. }
$$

Substituting (14) into the correction functionals (11) results in the following iteration formula:

$$
\begin{aligned}
& x_{1_{n+1}}(t)=x_{1_{n}}(t)-\int_{0}^{t}\left\{x_{1_{n}}^{\prime}(\xi)-\frac{38}{1180} x_{3_{n}}(\xi)-(1+\sin (\xi))\right. \\
& \left.+\frac{38}{2900} x_{1_{n}}(\xi)\right\} d \xi, \\
& x_{2_{n+1}}(t)=x_{2_{n}}(t)-\int_{0}^{t}\left\{x_{2_{n}}^{\prime}(\xi)-\frac{18}{2900} x_{1_{n}}(\xi)\right. \\
& \left.+\frac{18}{850} x_{2_{n}}(\xi)\right\} d \xi \\
& x_{3_{n+1}}(t)=x_{3_{n}}(t)-\int_{0}^{t}\left\{x_{3_{n}}^{\prime}(\xi)-\frac{20}{2900} x_{1_{n}}(\xi)\right. \\
& \left.-\frac{18}{850} x_{2_{n}}(\xi)+\frac{38}{1180} x_{3_{n}}(\xi)\right\} d \xi .
\end{aligned}
$$

We start with initial approximations $x_{1_{0}}=x_{1}(0)=$ $0, x_{2_{0}}=x_{2}(0)=0, x_{3_{0}}=x_{3}(0)=0$.
By the above iteration formula, we can obtain a few first terms being calculated:

$$
\begin{aligned}
x_{1_{1}}= & 1+t-\cos (t), \\
x_{2_{1}}= & 0, \\
x_{3_{1}}= & 0, \\
x_{1_{2}}= & 1+\frac{1431}{1450} t-\cos (t)-\frac{19}{2900} t^{2}+\frac{19}{1450} \sin (t), \\
x_{2_{2}}= & \frac{9}{1450} t+\frac{9}{2900} t^{2}-\frac{9}{1450} \sin (t), \\
x_{3_{2}}= & \frac{1}{145} t+\frac{1}{290} t^{2}-\frac{1}{145} \sin (t), \\
x_{1_{3}}= & \frac{123998651}{124047500}+\frac{1431}{1450} t-\frac{123998651}{124047500} \cos (t) \\
& -\frac{1576601}{248095000} t^{2}+\frac{19}{1450} \sin (t)+\frac{16283}{248095000} t^{3}, \\
x_{2_{3}}= & \frac{9}{1450} t+\frac{42849}{14297000} t^{2}-\frac{9}{1450} \sin (t) \\
& +\frac{1521}{7148500}-\frac{507}{14297000} t^{3}-\frac{1521}{7148500} \cos (t), \\
x_{3_{3}}= & \frac{1}{145} t+\frac{7080881}{2108807500} t^{2}-\frac{1}{145} \sin (t) \\
& +\frac{190869}{1054403750}-\frac{63623}{2108807500} t^{3} \\
& -\frac{190869}{1054403750} \cos (t) .
\end{aligned}
$$

$\vdots$

Continuing in this manner, we can find the rest of components.

A few $m$ terms approximation to the solutions are considered as

$$
x_{1}(t) \approx x_{1_{m}}, \quad x_{2}(t) \approx x_{2_{m}}, \quad x_{3}(t) \approx x_{3_{m}} .
$$

Some numerical values of solutions are presented in Table 1 with $n$ being the order of iteration formula of VIM.

3.2. Impulse Input. The impulse input model is used for pollutants that have been released into the lake immediately. Impulse input functions have a spike, and everywhere else the function is zero. The spike represents the time at which the contaminant was dumped.

An example would be dumping one barrel of gasoline into a lake at time zero. In one short heavy dumping of waste, with no reaction with the lake, input $p(t)$ is equal to 100 units for a duration of 10 units of time. 
TABLE 1: Result for sinusoidal model.

\begin{tabular}{ccccc}
\hline$n$ & $t$ & $x_{1}(t)$ & $x_{2}(t)$ & $x_{3}(t)$ \\
\hline 1 & 0.00 & 0.0000000 & 0.0000000 & 0.0000000 \\
2 & 0.01 & $0.1004934 \times 10^{-1}$ & $0.3113793 \times 10^{-6}$ & $0.3459770 \times 10^{-6}$ \\
3 & 0.02 & $0.2019735 \times 10^{-1}$ & $0.1249370 \times 10^{-5}$ & $0.1388263 \times 10^{-5}$ \\
4 & 0.03 & $0.3044401 \times 10^{-1}$ & $0.2820067 \times 10^{-5}$ & $0.3133661 \times 10^{-5}$ \\
5 & 0.04 & $0.4078927 \times 10^{-1}$ & $0.5029427 \times 10^{-5}$ & $0.5588849 \times 10^{-5}$ \\
6 & 0.05 & $0.5123310 \times 10^{-1}$ & $0.7883429 \times 10^{-5}$ & $0.8760533 \times 10^{-5}$ \\
7 & 0.06 & $0.6177546 \times 10^{-1}$ & $0.1138805 \times 10^{-4}$ & $0.1265541 \times 10^{-4}$ \\
8 & 0.07 & $0.7241617 \times 10^{-1}$ & $0.1554927 \times 10^{-4}$ & $0.1728018 \times 10^{-4}$ \\
9 & 0.08 & $0.8315528 \times 10^{-1}$ & $0.2037305 \times 10^{-4}$ & $0.2264153 \times 10^{-4}$ \\
10 & 0.09 & $0.9399265 \times 10^{-1}$ & $0.2586535 \times 10^{-4}$ & $0.2874615 \times 10^{-4}$ \\
11 & 0.10 & 0.1049282 & $0.3203213 \times 10^{-4}$ & $0.3560070 \times 10^{-4}$ \\
\hline
\end{tabular}

Now we assume $p(t)=100$, and so the system (6) with parameters that are defined in Section 3.1 will be

$$
\begin{gathered}
x_{1}^{\prime}(t)=\frac{38}{1180} x_{3}(t)+100-\frac{38}{2900} x_{1}(t), \\
x_{2}^{\prime}(t)=\frac{18}{2900} x_{1}(t)-\frac{18}{850} x_{2}(t), \\
x_{3}^{\prime}(t)=\frac{20}{2900} x_{1}(t)+\frac{18}{850} x_{2}(t)-\frac{38}{1180} x_{3}(t), \\
x_{1}(0)=0, \quad x_{2}(0)=0, \quad x_{3}(0)=0 .
\end{gathered}
$$

Therefore, by solving system (18) with VIM, as have been shown in Sinusoidal input model, the following results are earned:

$$
\begin{aligned}
& x_{1_{1}}=100 t, \quad x_{2_{1}}=0, \quad x_{3_{1}}=0 \\
& x_{1_{2}}=100 t-\frac{19}{29} t^{2} \\
& x_{2_{2}}=\frac{9}{29} t^{2}, \quad x_{3_{2}}=\frac{10}{29} t^{2}, \\
& x_{1_{3}}=100 t-\frac{19}{29} t^{2}+\frac{16283}{2480950} t^{3} \\
& x_{2_{3}}=\frac{9}{29} t^{2}-\frac{507}{142970} t^{3} \\
& x_{3_{3}}=\frac{10}{29} t^{2}-\frac{63623}{21088075} t^{3} .
\end{aligned}
$$

Some numerical values of solutions are presented in Table 2 with $n$ being the order of iteration formula of VIM.

3.3. Step Model. The step input model is used for pollutants that enter the lake at steady concentration and rate and continue the same way indefinitely. The pollutant enters the system at time zero, and the concentration previous to time zero was nothing. The significant points for step input are that the input increases suddenly at time zero and that the input remains essentially constant after time zero.
An example would be when a manufacturing plant starts production at time zero and starts dumping raw sewage at a constant rate and concentration.

Now we assume $p(t)=100 t$, so system (6) with parameters that are defined in Section 3.1 would be

$$
\begin{gathered}
x_{1}^{\prime}(t)=\frac{38}{1180} x_{3}(t)+100 t-\frac{38}{2900} x_{1}(t), \\
x_{2}^{\prime}(t)=\frac{18}{2900} x_{1}(t)-\frac{18}{850} x_{2}(t), \\
x_{3}^{\prime}(t)=\frac{20}{2900} x_{1}(t)+\frac{18}{850} x_{2}(t)-\frac{38}{1180} x_{3}(t), \\
x_{1}(0)=0, \quad x_{2}(0)=0, \quad x_{3}(0)=0 .
\end{gathered}
$$

Now by using VIM for system (20), the following approximations for the solution are earned:

$$
\begin{aligned}
& x_{1_{1}}=50 t^{2}, \\
& x_{2_{1}}=0, \\
& x_{3_{1}}=0 \\
& x_{1_{2}}=50 t^{2}-\frac{19}{87} t^{3}, \\
& x_{2_{2}}=\frac{3}{29} t^{3}, \\
& x_{3_{2}}=\frac{10}{87} t^{3}, \\
& x_{1_{3}}=50 t^{2}-\frac{19}{87} t^{3}+\frac{16283}{9923800} t^{4}, \\
& x_{2_{3}}=\frac{3}{29} t^{3}-\frac{507}{571880} t^{4}, \\
& x_{3_{3}}=\frac{10}{87} t^{3}-\frac{63623}{84352300} t^{4} .
\end{aligned}
$$


TABLE 2: Result for impulse model.

\begin{tabular}{ccccc}
\hline$n$ & $t$ & $x_{1}(t)$ & $x_{2}(t)$ & $x_{3}(t)$ \\
\hline 1 & 0.00 & 0.0000000 & 0.0000000 & 0.0000000 \\
2 & 0.01 & 0.9989345 & $0.3103448 \times 10^{-4}$ & $0.3448275 \times 10^{-4}$ \\
3 & 0.02 & $0.1999738 \times 10^{1}$ & $0.1241095 \times 10^{-3}$ & $0.1379069 \times 10^{-3}$ \\
4 & 0.03 & $0.2999411 \times 10^{1}$ & $0.2792146 \times 10^{-3}$ & $0.3102634 \times 10^{-3}$ \\
5 & 0.04 & $0.3998952 \times 10^{1}$ & $0.4963248 \times 10^{-3}$ & $0.5515311 \times 10^{-3}$ \\
6 & 0.05 & $0.4998363 \times 10^{1}$ & $0.7754190 \times 10^{-3}$ & $0.8616919 \times 10^{-3}$ \\
7 & 0.06 & $0.5997643 \times 10^{1}$ & $0.1116476 \times 10^{-2}$ & $0.1240728 \times 10^{-2}$ \\
8 & 0.07 & $0.6996792 \times 10^{1}$ & $0.1519474 \times 10^{-2}$ & $0.1688621 \times 10^{-2}$ \\
9 & 0.08 & $0.7995810 \times 10^{1}$ & $0.1984392 \times 10^{-2}$ & $0.2205353 \times 10^{-2}$ \\
10 & 0.09 & $0.8994698 \times 10^{1}$ & $0.2511210 \times 10^{-2}$ & $0.2790905 \times 10^{-2}$ \\
11 & 0.10 & $0.9993455 \times 10^{1}$ & $0.3099905 \times 10^{-2}$ & $0.3445261 \times 10^{-2}$ \\
\hline
\end{tabular}

TABle 3: Result for step model.

\begin{tabular}{ccccc}
\hline$n$ & $t$ & $x_{1}(t)$ & $x_{2}(t)$ & $x_{3}(t)$ \\
\hline 1 & 0.00 & 0.0000000 & 0.0000000 & 0.0000000 \\
2 & 0.01 & $0.4999782 \times 10^{-2}$ & $0.1034448 \times 10^{-6}$ & $0.1149425 \times 10^{-6}$ \\
3 & 0.02 & $0.1999825 \times 10^{-1}$ & $0.8274444 \times 10^{-5}$ & $0.9194195 \times 10^{-6}$ \\
4 & 0.03 & $0.4499410 \times 10^{-1}$ & $0.2792385 \times 10^{-5}$ & $0.3102837 \times 10^{-5}$ \\
5 & 0.04 & $0.7998603 \times 10^{-1}$ & $0.6618421 \times 10^{-5}$ & $0.7354391 \times 10^{-5}$ \\
6 & 0.05 & 0.1249727 & $0.1292549 \times 10^{-4}$ & $0.1436103 \times 10^{-4}$ \\
7 & 0.06 & 0.1799528 & $0.2233334 \times 10^{-4}$ & $0.2481781 \times 10^{-4}$ \\
8 & 0.07 & 0.2449251 & $0.3546148 \times 10^{-4}$ & $0.3940718 \times 10^{-4}$ \\
9 & 0.08 & 0.3198882 & $0.5292922 \times 10^{-4}$ & $0.5881969 \times 10^{-4}$ \\
10 & 0.09 & 0.4048409 & $0.7535566 \times 10^{-4}$ & $0.8374364 \times 10^{-4}$ \\
11 & 0.10 & 0.4997818 & $0.1033597 \times 10^{-3}$ & $0.1148671 \times 10^{-3}$ \\
\hline
\end{tabular}

Some numerical values of solutions are presented in Table 3 with $n$ being the order of iteration formula of VIM.

\section{Conclusion and Discussion}

In this paper, the problem of pollutions of three lakes with interconnecting channels has been considered. Different input models have been used for monitoring the pollution in three lakes. The variational iteration method as a powerful device has been used to solve the system of differential equations, governing on the problem. For different input models, the results of applying $n$ times iteration formula in VIM procedure for different times $t$ (year) are shown in suitable tables. The results are compared with those obtained by ADM in [11]. This comparison shows that the results are the same, but the solution procedure of the VIM is simpler than of ADM and the amounts of computations required in VIM is much lesser than ADM.

\section{Acknowledgment}

The authors are grateful to the anonymous reviewer for his (her) useful comments which have improved the quality of the paper.

\section{References}

[1] J.-H. He, "Variational iteration method-a kind of non-linear analytical technique: some examples," International Journal of Non-Linear Mechanics, vol. 34, no. 4, pp. 699-708, 1999.

[2] J.-H. He, "Some asymptotic methods for strongly nonlinear equations," International Journal of Modern Physics B, vol. 20, no. 10, pp. 1141-1199, 2006.

[3] J. H. He, Non-Perturbative Methods for Strongly Nonlinear Problems, Dissertation, de-Verlag in Internet $\mathrm{GmbH}$, Berlin, Germany, 2006.

[4] J. Biazar and H. Ghazvini, "He's variational iteration method for solving hyperbolic differential equations," International Journal of Nonlinear Sciences and Numerical Simulation, vol. 8, no. 3, pp. 311-314, 2007.

[5] J. Biazar and H. Ghazvini, "An analytical approximation to the solution of a wave equation by a variational iteration method," Applied Mathematics Letters, vol. 21, no. 8, pp. 780-785, 2008.

[6] D. D. Ganji, M. Nourollahi, and E. Mohseni, "Application of He's methods to nonlinear chemistry problems," Computers and Mathematics with Applications, vol. 54, no. 7-8, pp. 11221132, 2007.

[7] S. Abbasbandy, "A new application of He's variational iteration method for quadratic Riccati differential equation by using Adomian's polynomials," Journal of Computational and Applied Mathematics, vol. 207, no. 1, pp. 59-63, 2007.

[8] M. Inokuti, et al., "General use of the Lagrange multiplier in non-linear mathematical physics," in Variational Method in 
the Mechanics of Solids, S. Nemat-Nasser, Ed., pp. 156-162, Pergamon Press, Oxford, UK, 1978.

[9] J. H. He, "Semi-inverse method of establishing generalized principles for fluid mechanics with emphasis on turbo machinery aerodynamics," International Journal of Turbo JetEngines, vol. 14, no. 1, pp. 23-28, 1997.

[10] B. A. Finlayson, The Method of Weighted Residuals and Variational Principles, Academic Press, New York, NY, USA, 1972.

[11] J. Biazar, L. Farrokhi, and M. R. Islam, "Modeling the pollution of a system of lakes," Applied Mathematics and Computation, vol. 178, no. 2, pp. 423-430, 2006. 

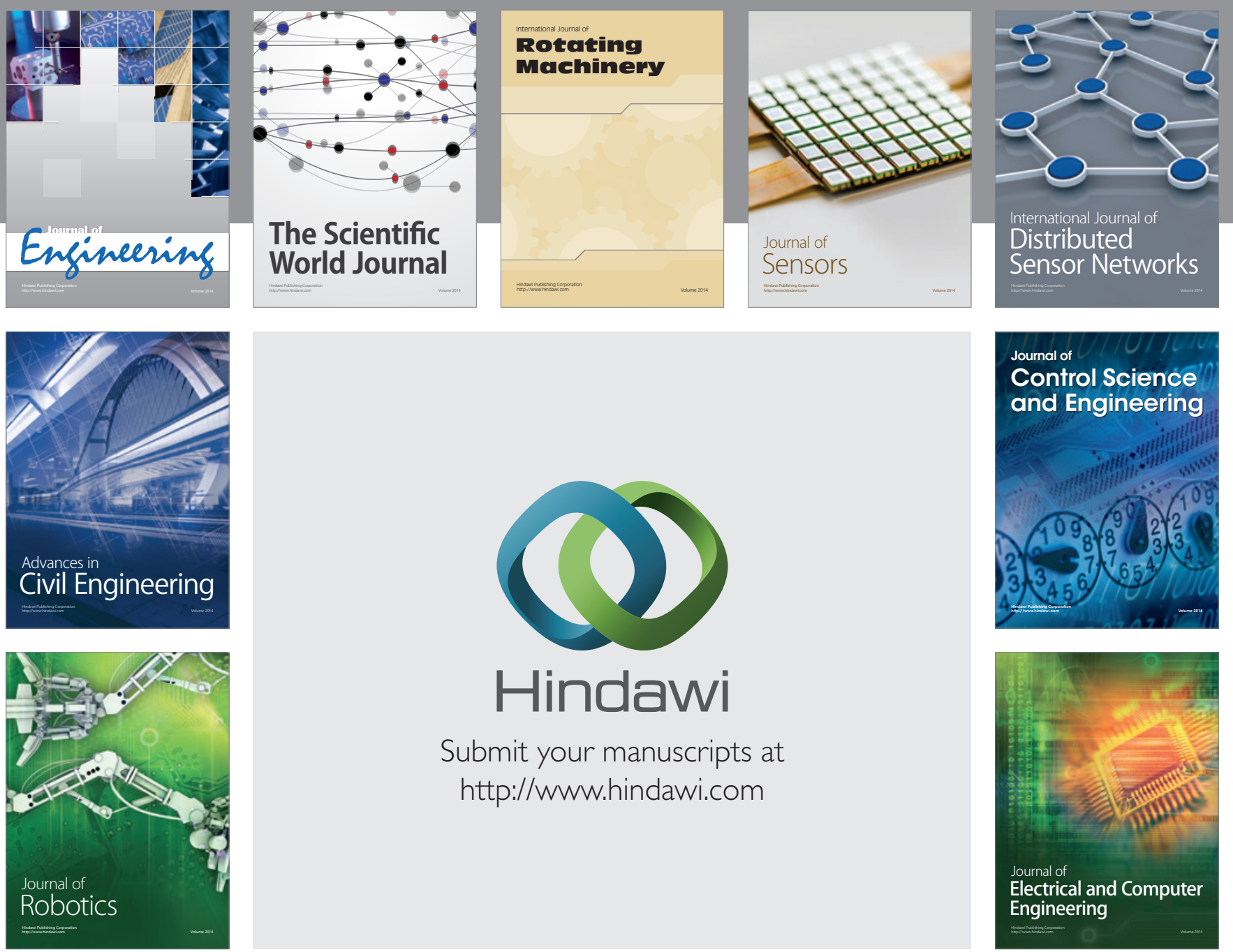

Submit your manuscripts at

http://www.hindawi.com
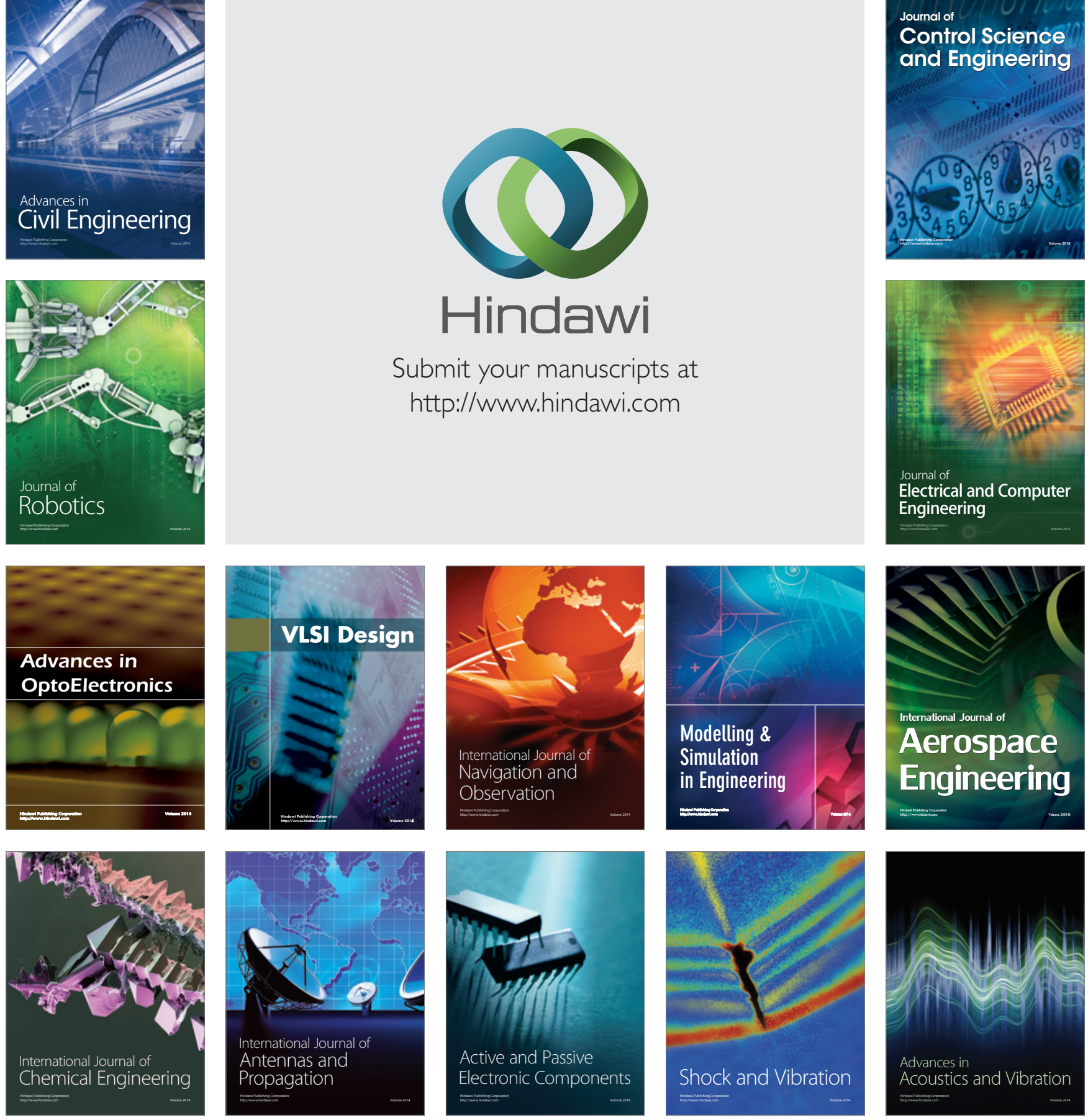